\title{
Utopies et angoisses de l'entre-deux identitaire chez les exilés/migrants africains : La Traversée nocturne d'Isaac Bazié
}

\author{
Par Alain Cyr Pangop Kameni \\ Université de Saarbrücken (Allemagne)/ Université de Dschang (Cameroun)
}

\section{Introduction}

On peut remonter à Thomas More pour comprendre pourquoi immigration, exil, hybridité culturelle et écriture de la glocalisation ${ }^{1}$ entrent en collusion avec l'utopie aujourd'hui dans la littérature francophone. En effet, Grand chancelier d'Angleterre, Thomas More s'était engagé à une satire sociale des pays d'Europe en général et de la société anglaise en particulier, sous le règne d'Henri VIII. Dans son œuvre polémique, il décrit la misère des populations tant rurales qu'urbaines, acculées à se livrer au banditisme sévèrement réprimé par l'Etat, par de nobles désœuvrés qui détiennent l'essentiel des terres transformées en pâturage, au détriment des paysans. Trois axes majeurs guident la démarche utopique chez cet auteur : l'entrée en conflit avec la réalité, la création d'un espace fictionnel et la réflexion sur cet univers imaginaire. Parce qu'il part d'une prise de conscience des tares de la société et qu'il entre en conflit avec le système en place, l'utopiste ne peut être défini dans l'absolu comme un insensé qui se livre à des rêveries chimériques faisant table rase de la réalité, comme peuvent le laisser penser plusieurs dictionnaires. Mais cela ne constitue que le versant négatif de l'imaginaire de l'utopie. Le système de la machine utopique, l'autre versant, positif, relève d'un voyage imaginaire qui décrit un monde aux lois inacceptables et donc à déconstruire. Les propositions découlant de la spéculation sur un univers fictif organisent la création littéraire chez les utopistes et s'étend à divers domaines tels que le travail, la religion, les traditions, les guerres, l'éducation, l'économie, etc. Le privilège de la spéculation par rapport à l'action ne saurait faire perdre de vue ici le caractère résolument subversif et compensatoire à l'impuissance d'agir de la méthode utopique. C'est également le reflet de cette exploration du possible que dégagent les productions romanesques des exilés/ migrants africains qui font face aux élites locales frappées de cécité car, "un fatal bandeau les aveugle, le bandeau des préjugés et les faux principes dont on les a pétris et infectés dès l'enfance » (More 93).

Sous ce rapport, la littérature africaine francophone vit, se produit, se distribue et se lit majoritairement en exil. Et si cet exil constitue pour certains écrivains un asile

\footnotetext{
${ }^{1}$ Nous employons ce terme ici pour désigner le jeu mondial de la diffusion à l'échelle planétaire qui se confronte aux localités, tout en englobant des espaces rêvés.
} 
politique ou une véritable expatriation, elle devient pour d'autres une stratégie littéraire, car considérant leur pays d'origine comme « désert culturel », ils le quittent librement pour d'autres horizons qui leur offrent plus de possibilités (Ranvier 205207). Mais cette émigration - volontaire ou involontaire - est souvent vécue dans le pays d'accueil à la fois comme un enrichissement et un conflit culturels. Car dans la mobilité, "territorialisation et déplacement » (Imbert 101), les écrivains en arrivent parfois jusqu'au nomadisme littéraire ${ }^{2}$, dans la mesure où ils évoluent entre plusieurs pays, plusieurs langues et plusieurs cultures.

C'est cette situation qui, à l'origine, amène la diaspora noire à produire une nouvelle forme $d^{\prime}$ " écriture décentrée ${ }^{3}$ que traduit le concept de migritude ${ }^{4}$. Originaires des pays décolonisés, les auteurs africains de l'immigration traitent majoritairement de sujets postcoloniaux ${ }^{5}$. Christiane Albert (2005) a eu à démontrer, à la suite d'autres critiques, le poids littéraire de ces auteurs. Émile Ollivier va plus loin, avec le concept de « Migrance » qui prend un poids plus consistant :

Migrer à n'en pas douter est une tragédie, mais c'est aussi un salut. Il faut essayer de faire avec ces deux versants, et l'on se trompe soi-même si on en oublie un. J'ai forgé le mot migrance pour indiquer que la migration est une douleur, une souffrance (la perte des racines, d'une certaine «naturalité ») et, en même temps, une posture de distance, un lieu de vigilance. Je vois très bien les pertes que cette situation inflige : le bain utérin, la langue maternelle, le sol, l'éclatement de l'identité, mais dans le même temps, il y a une contrepartie à cette violence et à cette brutalité, celle d'une individualité polyphonique, celle de naître à un univers décloisonné qui est irisation, rhizome, foisonnement, bourgeonnement de vie et de liberté. (Ollivier 26)

On peut observer ici que la rencontre avec l'autre va générer deux attitudes différentes face au paradigme généalogique. $D^{\prime}$ un côté, des réactions d'autoprotection, et de l'autre, des mouvements d'hybridation identitaire bâtis sur l'effacement/actualisation de l'origine. Si l'on s'en tient au défi de la quête de légitimité, le conditionnement extralittéraire y a une place primordiale, au regard des moyens déployés par les écrivains dans leurs efforts de s'émanciper de la tendance à

\footnotetext{
${ }^{2}$ La dualité culturelle fait de l'écrivain francophone africain un sujet nomade, un être périphérique enjambant des frontières. «In between» selon l'appellation de la critique anglo-saxonne, les écrivains migrants postcoloniaux sont sujets à la transhumance, relativement à leur absence d'ancrage personnel. C'est peut-être pourquoi leur écriture se décline sous des modalités pluriculturelles.

${ }^{3}$ Pour plus de lumière sur cette expression, voir le collectif dirigé par Michel Laronde (1997).

${ }^{4}$ Ce concept développé par Jacques Chevrier (2006) vient appuyer l'affirmation d'une pluralité de référents et la remise en cause des frontières littéraires, dans la mesure où on se demande si c'est le lieu de naissance, de résidence ou alors l'influence de la culture d'origine qui détermine l'identité littéraire d'un écrivain.

${ }^{5}$ La coexistence de divers univers symboliques hérités de la colonisation et renforcé par le contexte de la mondialisation oblige l'écrivain africain contemporain à aspirer à se situer dans le champ littéraire mondial, tout en prenant pied dans un ensemble socioculturel africain souffrant souvent du mépris du regard occidental. Aussi, à l'inverse des discours stéréotypés se lit-il un discours sur l'entre-deux insécurisant que les théories postcoloniales de Jean Marc Moura (1999) et de Homi Bhabha (2007) ont eu à problématiser.
} 
la catégorisation opérée par des institutions littéraires. En tenant compte du contexte d'émergence et de la forme de cette littérature de l'entre-deux, nous tachons ici de présenter l'exemple de La Traversée nocturne ${ }^{6}$ d'Isaac Bazié, plus connu comme critique que comme auteur. En investissant les territoires de l'entre-deux, la quête d'identité $^{7}$ achoppe sur les stéréotypes dans la création d'identités nouvelles dans le récit de $L T N$ Ce texte, peu analysé par la critique, nous donne l'opportunité d'examiner le profil thématique et idéologique du roman francophone africain issu de l'immigration; dans le sens où LTN dit à la fois les difficultés de l'émigration, à la suite de Cheik Hamidou Kane, Alain Mabanckou et Fatou Diome, et celles de la vie au pays, dans le sillage de la critique sociale qui sous-tend le roman africain.

\section{Utopie, contre-utopie et éclatement identitaire}

La notion de l'entre-deux identitaire résiste aux définitions univoques, sans doute parce qu'elle caractérise, de par sa nature, l'insaisissable, l'indicible, ce qui est présent et voilé à la fois. Au fond, l'entre-deux identitaire échappe à toute systématisation. Il constitue un choix de l'instabilité, conformément au Dictionnaire international des termes littéraires qui le voit comme «ce qui n'est pas décidé, [...] ce qui ne peut être défini dans l'opposition des contraires ou des différences» («entre-deux»). Les valeurs habituellement associées à la postmodernité, à l'hétérogénéité, à la juxtaposition, à la fragmentation, au pluralisme, à la non-hiérarchisation, etc, lui correspondent comme un gant. L'entre-deux identitaire, c'est aussi le passage - l'exil géographique faisant écho à l'exil intérieur - dans une langue autre que la langue maternelle. Comme le relève Yves Chemla dans le cadre de la littérature francophone:

Dire l'ailleurs, bien souvent, c'est aussi prendre le parti de la «migrance». A partir de ce moment décisif est rompu le lien à la tradition active. Il faut devenir autre sans cesser d'être soi, et parvenir dans ce paradoxe à nommer ce qui dans l'ailleurs contrevient à l'accomplissement de soi. Il faut parcourir un ailleurs dont la description est déjà épuisé par plusieurs siècles de littérature et dans une langue que, bien souvent, les personnagesnarrateurs maîtrisent mieux que les autochtones et les indigènes européens (Chemla 48)

Autrement dit, le dédoublement identitaire passe d'abord par l'adoption de la langue $\mathrm{du}$ pays d'accueil qui permet une distanciation, souvent salutaire, une réconciliation du passé et du présent. À l'échelle des fictions romanesques francophones d'Afrique, l'entre-deux identitaire est un espace créateur où se questionnent la rencontre des cultures et ses conséquences, l'euphorique et le dysphorique ; où l'on repousse sans

\footnotetext{
${ }^{6}$ Dans la suite du texte, les références à cette œuvre seront indiquées par le sigle $L T N$, suivi de la page placée entre parenthèses.

7 La notion d'identité viendrait de l'idée de ressemblance extrême: deux êtres sont identiques lorsqu'ils pourraient être substitués l'un par l'autre. Elle dévoile fragilité et marginalité en contexte de migration. Car étudier la migration comme forme d'identité culturelle revient à vérifier si chez les auteurs les questions traitées et les personnages ressemblent, ou mieux, s'identifient à ceux de la société de référence.
} 
cesse les limites entre le rêve et la réalité. Dans la quête de leur légitimité et la revendication de leur marginalité, les écrivains de la nouvelle diaspora ${ }^{8}$ vont progressivement et simultanément se désengager de leur culture d'origine et de la culture d'accueil.

Parti du Burkina Faso pour l'Allemagne ${ }^{9}, c^{\prime}$ est finalement en tant que résidant du Canada que le Burkinabé Isaac Bazié décrit son petit coin d'Afrique. Ce faisant, son texte ne s'adresse pas directement aux lecteurs africains seulement, mais à tous les lecteurs francophones du monde entier. Allant au-delà du label du sujet colonial, Bazié insiste sur l'identité hybride et articule avec insistance les revendications d'acceptation. Prenant distance par rapport au discours sur l'ethnicité, il nie l'insertion dans la catégorie des littératures de la minorité. Déjà, ses réflexions critiques antérieures défendent ces lignes de démarcation. La perspective qu'il développe dans ce sens s'éloigne de manière critique des discours apologétiques sur la transitivité comprise comme nomadisme sans attaches et sans fin, errance de sujets aux compétences interculturelles à l'épreuve de toute barrière. Bazié est au fait de l'instabilité existentielle du sujet notamment africain, pris dans l'écart de ce que Ambroise Kom (2000) a identifié comme l'héritage postcolonial, et les défis d'une hypermodernité dont il est plus que le passif spectateur. Par contre, il resitue le sujet dans un lieu de stabilité minimale, qui exige au plan de la prise de parole - quelle qu'elle soit - que celui-ci ait un lieu, une situation d'énonciation. Cette situation est le point d'ancrage à partir duquel le sujet de la traversée observe son univers et produit des énoncés qui l'y situent ou l'en éloignent. Aux yeux de Bazié (2004), il faut donc dire que si la traversée n'a pas de fin, elle a à tout le moins et de manière essentielle à son déploiement, des étapes, des situations qui justement, même précaires, ponctuelles et sous une forme symboliques, restent des moments privilégiés d'un positionnement et d'une renégociation des modalités d'un être au monde.

En termes de migration, il faut dire qu'à la différence de textes comme Dossier classé d'Henri Lopes par exemple, l'espace narratif de LTN et les parcours des personnages se situent dans le contexte africain, mais avec des structures parfaitement identifiables à celles sous-jacentes au profil migratoire que l'on retrouve par ailleurs et qui se dessinent sur l'axe Afrique-Europe, voire l'Amérique.

Subséquemment, la migration entraîne un métissage qui, lui-même génère des marginalités, dès lors qu'il y a des transformations impliquées par les interactions

\footnotetext{
8 L'expression «nouvelle diaspora» utilisée ici renvoie aux écrivains africains expatriés de la deuxième génération, c'est-à-dire ceux qui se sont mis à l'abri des dictatures féroces issues des indépendances officielles pour se livrer à la création littéraire. Abdourahmane Waberi les appelera les « enfants de la postcolonie ».

${ }_{9}^{9}$ Notons que Bazié a fait ses études au Burkina Faso et en Allemagne où on n'insiste pas trop sur la dénomination de l'origine des auteurs.
} 
communautaires. Dans ce sens, le système binaire sagesse africaine/rationalité occidentale s'estompe dans un nouvel espace identitaire qui combine les deux: expression d'utopie plurielle, c'est-à-dire le double rêve de contribuer à deux mondes différents sans appartenir ni à l'un ni à l'autre. LTN présente un tel tableau d'éclatement identitaire : l'initiation rituelle, la forêt sacrée, le port du masque à Katena ; la fabrique à l'occidentale, les automobiles, l'argent, le bureau, l'électricité et l'université à Wona comme lieu par excellence d'échange culturel.

Le fatalisme qu'on observe à travers un réel figé peut faire basculer l'utopie en contre-utopie $^{10}$ : d'où des angoisses identitaires, c'est-à-dire l'oscillation entre marginalité et désir d'une reconnaissance transculturelle ${ }^{11}$. Car, même si la problématique sociale et politique est évidente, l'accent est mis sur le déplacement. Sélom Komlan Gbanou notait déjà récemment que

Dans les Afriques de la postcolonie, l'œuvre littéraire, surtout celle des écrivains confrontés aux réalités de l'ailleurs européen pour cause de migration, fonctionne comme la mémoire d'un chaos extérieur et intérieur, et met en mouvement un mécanisme de représentation, une scénographie du réel qui n'incite pas à remanier le désordre sociopolitique, mais à le simuler, du moins scripturairement, en vue de créer une « intertextualité » plus ou moins consciente entre le réel et le fictionnel. (Gbanou 84)

En fait, la «délocalisation $»^{12}$ de l'individu engendre des transformations qui peuvent aller jusqu'à une dépersonnalisation, c'est-à-dire l'expérience d'un sentiment de perte de sens de la réalité dont le cas extrême est la dysphorie. Cette dépersonnalisation à son tour accorde au projet littéraire une reconquête tourmentée des identités ainsi déstabilisées. D'où des utopies désespérées, c'est-à-dire des rêves contrariés qui suscitent cette interpellation de Chemla : «Il y a désormais urgence à fabriquer du lien, à reconnaître la part de l'autre, à assumer la diversité des identités culturelles et à mettre en œuvre des solidarités efficaces sans occulter les vomissures de l'histoire. » (Chemla 53). Il convient maintenant d'analyser ces considérations à la lumière du récit romanesque.

\section{Utopies et métamorphoses identitaires dans le récit romanesque}

L'écriture de l'entre-deux identitaire associe des éléments, crée des liens, juxtapose des réalités, dont l'intertextualité sous toutes ses formes - citation, allusion, parodie, etc, - en est exemplaire ici. En fait, LTN est une juxtaposition de trois grands récits imbriqués: le premier, contemporain et urbain narre la relation de la famille de

\footnotetext{
${ }^{10}$ L'intégration d'un exilé/migrant à un nouveau milieu, une nouvelle langue, une nouvelle culture, peut-être rude et traumatisante. Elle est souvent synonyme de dépossession, de pertes de repères identitaires par l'acculturation et l'assimilation. Lire à ce sujet Alain Pangop (105-122).

${ }^{11}$ On peut consulter à ce sujet Jacques Derrida (1996) qui parle de « trouble de l'identité ».

${ }^{12}$ Ce terme emprunté à la géographie économique métaphorise ici le transfert d'une personne d'une localité à une autre, avec tout son héritage socioculturel, et parfois économique.
} 
Vourma avec la fabrique industrielle de la ville de Wona; le deuxième, rural et traditionnel, présente le rite d'initiation perturbé par Baki et Elema à Katena; le troisième qui se rapporte à la génération antérieure a trait à la naissance d'un enfant déjà circoncis dans la famille de Vourbié à Kiekiena. La construction tridimensionnelle du récit et le symbolisme qui s'y dégage laisse apparaître trois signes de l'unité Père-Fils-Saint-Esprit ${ }^{13}$. Cette logique trinitaire montre qu'on a affaire à l'unité dans la diversité des cultures. Autrement dit, dans LTN la nonlinéarité du récit et la fragmentation de la narration illustre une volonté d'embrasser à la fois le passé et le présent, le réel et l'imaginaire, l'histoire de l'Afrique contemporaine et celle de l'Occident. Le récit est composé de personnages en transit permanent dans un «entre-deux»: entre des désirs d'engagement pour la reconnaissance de la marginalité et une position d'accommodation à la conformité. En effet, Bazié retravaille justement la notion de transitivité de manière radicale, en la redéployant sur trois niveaux : géographique, textuel et identitaire.

Au niveau géographique, Vourbié qui doit passer le fleuve avec sa famille du fait de sa désobéissance est considéré comme mort à l'instar de sa femme Elema :

Nous ne les connaissons plus. Ils sont morts aujourd'hui. Ils iront loin, là où personne de nous ne court le risque de les rencontrer. Ils s'établiront où ils veulent, mais loin de notre région. Si l'un d'entre vous venait à les rencontrer, il ne s'arrêtera point, ne saluera point et ne partagera point la même ombre qu'eux. Il ne se retournera point pour les voir s'éloigner et il veillera à éviter tout contact avec eux, par crainte d'être lui-même exposé à la peine de mort. Celui qui leur offrira son hospitalité ne vivra pas après leur départ assez longtemps encore pour répéter le geste sacrilège. (Bazié, LTN, 173-174)

Pareillement, lorsque le personnage d'Edmond quitte la maison parentale pour aller vivre chez Alice, il signe l'acte de décès de l'adolescence dans sa vie et affronte la responsabilité de son destin qu'il compte prendre en main. Aussi, la disparition de Baki, ce vieux combattant de l'époque coloniale, marque-t-elle l'aporie de la tradition, le courage de faire un saut dans l'inconnu (nous y reviendrons). L'escapisme - cette attitude qui consiste à se retirer du monde et de la vie civique, par fuite ou désabusement, par opposition à la prise de parole ou à l'action individuelle ou sociale - chez Vourbié, Edmond et Baki les conduit vers une identité de substitution, une émigration empreinte d'exil forcé qui justifie la métaphore nocturne contenue dans le titre du roman.

Au niveau textuel, les intertextes manifestent la transitivité. En fait, les pratiques intertextuelles dans $L T N$ se déclinent sous plusieurs formes : elles sont soit explicites, soit implicites. Elles sont aussi littéraires, bibliques et mythologiques. Du point de vue de l'explicite, on observe dans LTN des intertextes littéraires prenant les contours

\footnotetext{
${ }_{13}$ Notons que l'utilisation par l'homme de trois formes complémentaires de la symbolisation est généralement perçue comme formulation mythique dans la culture chrétienne, sous la forme du mystère de la Sainte Trinité. Dieu est un en trois personnes : le Père, le Fils et Saint-Esprit.
} 
de la parodie. Par exemple, s'agissant des ventripotents de Wona, on lit : « Mangez et prenez de la graisse, vous ferez le bonheur des termites » (Bazié, LTN 100). Il s'agit ici de la parodie $\mathrm{du}$ "Laboureur et ses enfants » extrait bien connu des Fables de la Fontaine : «Travaillez, prenez de la peine, c'est le fond qui manque le moins ».

De même, il est fait référence à l'histoire grecque antique : l'épée de Damoclès (Bazié, LTN 217). Lorsque LTN évoque la pancarte où il est écrit en noir : " malheur à ceux qui bâillonnent leurs peuples!» (Bazié, LTN 99), il est bien question d'un intertexte pictural significatif. Cet énoncé intertextuel fait en même temps du texte littéraire un écho percutant du discours social et politique dans le contexte de la révolution au Burkina Faso (1984-1987) avec, en avant-scène, le glas sonnant depuis les bas-fonds $\mathrm{du}$ règne des potentats. En outre, LTN fait plus usage de l'intertexte implicite. Par exemple, il est dit d'Elema, la femme du vieux Baki qu' " elle savait que cette heure décisive arriverait » (Bazié LTN 75). Ce qui laisse penser à l'arrestation de Jésus dans le jardin au mont des oliviers alors qu'Il priait avec ses disciples. Pareillement, le discours tenu lors de la répudiation de Vourbié et de sa famille nous fait savoir que : «L'ancien, qui avait jusqu'à présent parlé à l'adresse de Vourbié, se retourna maintenant vers la communauté que l'on avait rassemblée et qui était aussi assise à même le sol. Plusieurs centaines de femmes, d'enfants et d'hommes silencieux. » (Bazié LTN 173). N'est-ce pas là un intertexte biblique relatif à la foule que Jésus fit asseoir à même le sol ? De plus, s'agissant toujours de Vourbié, on peut lire qu' "Un homme marche à la tête d'une colonne d'hommes, de femmes et d'enfants » (Bazié LTN 202). Cela ressemble étrangement à Jésus suivi de la foule. On peut aller plus loin pour voir que Vourbié se déplace comme Jésus : «Lui et les siens traversent le fleuve» (Bazié LTN 203). Cette traversée est similaire à celle de Josué dans Les Saintes Écritures ou encore à celle de Moïse et les siens, pour échapper à Pharaon qui les poursuivait. Toutefois, l'implicite dans l'intertextualité telle qu'elle apparaît dans LTN n'est pas seulement de nature biblique. Elle est aussi de nature mythologique. La déclaration d'Edmond nous rappelle le mythe de Sisyphe condamné à rouler éternellement la pierre au-dessus de la colline : «C'est toujours la même histoire (...) ça ne finira donc jamais » (Bazié LTN 84)

La force de ces pratiques intertextuelles réside en leurs significations respectives. L'intertexte biblique recèle une force de consolation face à la situation qui se vit à Wona, en pensant à la souffrance du Christ et de ses prophètes. La parodie du «laboureur et ses enfants » de La Fontaine renvoie aux hauts cadres de Wona tels que Drabo, le chef de la fabrique, le Capitaine démocrate, et bien d'autres. Tous ceuxci constituent ce que le narrateur a appelé « les ventripotents ». La parodie sert donc de satire contre ceux qui spolient le petit peuple.

L'intertexte pictural connote le tragique du tableau, la mort qui guette les instigateurs de la discorde, de la hantise et de la désolation. Le caractère irréversible de la 
situation tragique qui se vit à Wona est mis en exergue par les intertextes mythologiques.

Qu'elles soient explicites ou implicites, les pratiques intertextuelles chez Bazié sont toujours littéraires, picturales, bibliques et mythologiques, pour témoigner de ce que chaque texte porte de manière plus ou moins visible, la trace et la mémoire d'un héritage. On le voit, l'intertextualité permet d'introduire la relation dialogique au niveau littéraire, par la convocation de textes, par l'allusion à d'autres œuvres et à d'autres cultures.

Deux univers culturels, deux imaginaires se rencontrent dans LTN par mise en abyme, pour porter un message. Autrement dit, les écrits de l'immigration «associent diverses cultures afin de se forger une identité construite de manière interne dans le texte littéraire. » (Feze 247) Défiant toute clôture, l'écrivain migrant «nourrit son récit de sa propre appartenance à une société construite, constituée et non innée. » (Feze 247)

Au niveau identitaire, on peut situer les enjeux de l'écriture de l'immigration au sein des cultures que traverse l'auteur, tout autant que ses personnages. Bien des pans du roman présentent métonymiquement et métaphoriquement la situation sociopolitique du Burkina Faso, ainsi que l'évolution culturelle sur trois générations. L'identité de l'exilé est ainsi mise à l'épreuve du jugement. L'homme au fusil est étranger chez lui. Être étranger chez soi est une situation paradoxale et peu confortable. L'expatriation devient alors une « ruse culturelle » en ce qui concerne les angoisses identitaires. Si Amin Maalouf qualifie les identités de «meurtrières » (Maalouf 1998), c'est-à-dire les risques de violence et d'intolérance inhérents à tout repli identitaire, c'est que l'identité oscille entre Ici et Ailleurs, dans une "glocalisation" de plus en plus affirmée, comme nous l'avons précédemment expliqué. À l'avenant, l'imaginaire composite de l'écrivain exilé/migrant participe de ce que Maingueneau appelle la «paratopie spatiale» (Maingueneau 72) pour signifier le perpétuel mouvement de la littérature liée au non-ancrage de l'écrivain.

Sous un autre rapport, le point de départ de l'hybridité culturelle est de l'ordre du mental dans la mesure où Vourma constate que les Africains eux-mêmes aspirent à être des Blancs : «Les Blancs à la peau noire ne se sont pas trompés de lieu où naître, eux-mêmes savent qu'ils ne sont pas des nôtres » (Bazié LTN 45). Cette assertion convoque à la mémoire Peau noire, masques blancs (1952) de Frantz Fanon, où il est question du complexe nègre à la confluence du colonialisme.

L'hybridité culturelle peut aussi se relever dans les noms des personnages qui évoluent dans le texte: Doogo, Vourma, Oli, Ovour, Ekio, Elema, Vourbié, etc tiennent de l'onomastique africaine, tandis que Paul, Edmond, Alice relèvent de la 
culture occidentale. Pareillement, Abou et Abdou relèvent de la religion musulmane. La forte présence musulmane est accentuée par ces propos du narrateur : «Les deux femmes allèrent choir dans un sourd vacarme (...) après des ablutions de nombreux musulmans de la concession » (Bazié LTN 27).

Ce brassage culturel peut aussi se lire dans la caractéristique de l'écriture. Les thèmes d'actualité du monde contemporain sont, à cet effet, présents dans le roman: corruption, violence, gabegie et détournements.

À cheval entre une écriture décalée et décentrée, les récits de la migritude sont caractérisés par le métissage culturel et la diversité linguistique. À la littérature orale, Isaac Bazié associe la littérature écrite. Les néologismes tels que « sans-espoir » (Bazié LTN 57), « no name» (Bazié LTN 59), etc, enrichissent linguistiquement et culturellement le texte, tout comme les déformations «Conzé teknik » (Bazié LTN, p. 72), « démoncratie » (Bazié LTN 137).

À l'hybridité culturelle engendrée par les cohabitations des personnages aux identités différentes, s'oppose la loi du silence imposée par la tradition orale ici ritualisée par l'initiation au sein de la forêt sacrée. Cette initiation instruit les initiés à ne jamais trahir le secret. Aussi, les stéréotypes et les interdits font-ils face aux autres formes de communication sociale issues des contrées lointaines dont le véhicule principal est l'école. "Les hommes qui ne traversent jamais le fleuve » affrontent ceux qui ont le courage de le faire et même ceux qui tentent de le faire en vain.

L'escapisme et la trans-spatialité constituent de ce point de vue, les conséquences d'un espoir fondé sur un horizon de l'ailleurs. Car la misère chez soi conduit nécessairement au rêve hyperbolique d'obtenir le bonheur chez l'autre. En outre, l'utopie littéraire $^{14}$ fonctionne dans LTN comme un mode caractérisation d'une constellation de rêves, de navigations dans des nuages d'imagination. Ce processus se décline en trois dimensions : l'illusion parentale, l'illusion juvénile et la plongée dans le passé15.

Tout part de l'espoir, du rêve hyperbolique du couple Vourma-Ekio à voir Edmond, leur unique fils encadré jusqu'à l'obtention de sa licence. Aussi Bazié fera-t-il dire au narrateur :

Edmond est soudain saisi par un sentiment de pitié pour cet homme et sa femme qui ont juré de scolariser leur fils unique, pour qu'il ne demeure pas aveugle et inutile dans une société où l'instruction est la clef du succès. Ses parents y ont cru. Pendant longtemps même. Mais plus le

\footnotetext{
${ }_{14}$ Sur ce sujet, consulter particulièrement Papa Samba Diop et Florence Paravy, Littératures africaines. Littératures francophones et utopies, Paris, Université Paris XII Val de Marnes, 2006.

15 Alors que l'utopie est une représentation magnifiée du réel en vue d'en dénoncer les travers, l'illusion consiste en une perception erronée de ce réel. Les illusions parentale et juvénile ici renvoient aux décalages d'appréciation du réel dans les deux classes d'âge.
} 
temps passe, plus ils se rendent compte que l'instruction seule, ne suffit pas pour réussir à Wona. (Bazié LTN 197)

Sacrifiant toute construction d'habitation pour investir dans les études de son fils, Vourma est aujourd'hui mis en congé technique et est proche de la retraite. Comme il est encore en location, la propriétaire du logement réclame un mois de loyer impayé et en viendra à une altercation verbale avec Ekio :

Tu me poses toutes ces questions à cause du loyer impayé de ce mois-ci ? Tu pourrais tout de même patienter un peu, nous n'avons jamais eu d'arriéré, et tu le sais.

- Hum ! Puisque tu préfères que nous parlions plutôt affaires, tu l'auras voulu. D'ailleurs je me demande ce que vous attendez pour me payer. Au fond, vos histoires ne m'intéressent pas. Mon argent, voilà ce que je veux! (Bazié LTN 24)

Pour avoir tout misé sur leur fils en rêvant de le voir réussir socialement, Vourma et son épouse sont aujourd'hui exposés aux injures, à l'humiliation.

Le rêve de voir réussir leur progéniture est ici au cœur de l'illusion parentale. Comme dans Le Ventre de l'Atlantique de Fatou Diome, l'émigré est toujours ce héros qui fait rêver de réussite sociale.

L'illusion juvénile prolonge celle parentale en ce sens que l'ensemble de la jeunesse rêve de victoires. En témoigne le cri de joie d'Edmond lorsqu'il réussit à son concours d'entrée à l'école normale. Cet espoir se dessine dans la conversation des camarades $\mathrm{du} \ll$ Pentagone $»:$

Alors, je vais te dire : moi Safi, quand je me marierai, ce ne sera pas pour rejoindre un homme dans sa maison pour qu'il me fasse des misères. Jamais! D'abord, je finis ma maîtrise, je me cherche le meilleur job de Wona, je m'installe correctement, et ce sera lui qui viendra chez moi. Le jour où il me casse les pieds, ouste!

- Ma sœur, s'il te plaît, parle pas fort, parce que s'il t'entend, il ne viendra même plus (...).

- Tais-toi et écoute. Le troisième groupe (des ventripotents). C'est vous et moi, les aspirants ventripotents. Le ventre, nous ne l'avons pas encore, mais croyez-moi, nous l'aurons. Il faut le vouloir seulement, après on verra comment $y$ arriver, ou bien Safi ?

- Et comment toi tu comptes y arriver ? Lui demanda, la jeune fille. Tu n'as même plus de bourse, tu ne sais pas pourquoi on t'a recruté comme enseignant sans que tu ne sois payé (Bazié LTN 58)

Wona en crise suscite, par la perte de son centre de gravité, la tension entre une résignation vouée à la mort et une mobilité, un déplacement salvateur.

$\mathrm{D}^{\prime}$ un tout autre point de vue LTN nous ramène trois générations en arrière pour faire rêver de la forme de vie de cette époque lointaine. En effet, une plongée dans le passé mène le lecteur à Katena où la transgression des lois expose à des sanctions extrêmes. Dans ce contexte où chacun sait à quoi s'en tenir, Baki est contraint à la disparition parce qu'il a choisi d'abattre «le masque », symbole du pouvoir traditionnel. 
De même, Elema est en proie à la mort parce qu'elle viole à plusieurs reprises les interdits en traversant le fleuve et en épiant le «masque » dans la forêt sacrée. Pour sa part, Vourbié est extradé du village pour n'avoir pas respecté les dernières volontés de son défunt père. Comme le relève Gbanou pour les métamorphoses du récit dans Le Pleurer-rire d'Henri Lopes: "Ces différents récits sont, plus que des digressions fantaisistes à fonction dilatoire, des romans en miniature dans le roman. Ils jouent sur la précarité et l'évanescence du réel soumis à des intrusions, des ruptures, des diffractions de toutes sortes. » (Gbanou 87).

Ainsi juxtaposées, on voit transparaître sous la plume de Bazié des récits qui portent une satire à la fois des impunités qui caractérisent la gouvernance contemporaine et de la rigidité du système traditionnel. Le déficit de gouvernance s'illustre dans LTN par la brutalité des policiers du Capitaine Démocrate qui répriment sévèrement les revendications estudiantines.

L'utopie permet ainsi à l'auteur de mettre en perspective les pratiques déshumanisantes du Capitaine Démocrate imposées par les puissances étrangères: dictature féroce, répressions brutales des grèves estudiantines, trucage des élections, pollution de l'environnement, etc.

Les profils de «La dame de fer » et de Drabo qui président successivement aux destinées de la Fabrique de Wona montrent le népotisme et le marchandage qui les portent à ce stade de la préséance sociale. Sous le couvert du sommet de l'État, les impérities de Drabo conduisent à la faillite de l'entreprise, dont la mise en congé technique de Vourma et de Doogo sans mesures compensatoires constitue les signes avant-coureurs.

À ce sombre tableau se greffent des crises multiformes que subit le peuple. À cause du licenciement de Vourma de son lieu de travail, son épouse Ekio non seulement aura une altercation avec sa logeuse, mais passera une nuit au commissariat pour échapper au viol par son beau-frère chez qui elle est allée solliciter de l'aide financière. Lorsqu'enfin Vourma perçoit ses frais de mise en congé technique, il est agressé par des malfrats et délesté de tous ses avoirs. Edmond, pour venger son père devenu ivrogne et adepte du P.M.U. (Pari Mutuel Urbain), ira se faire justice en arrachant miraculeusement des mains de Drabo, le paquet d'argent qu'il avait apprêté pour s'enfuir en Europe. Ce projet périlleux fait voler en éclat le contenu du sac, compliquant davantage le «vouloir vivre» du jeune Edmond qui a connu auparavant les déceptions dans les ministères, avec en prime une altercation avec le fils du « Capitaine Démocrate ».

Il se dévoile dans LTN un univers de la terreur comme pour justifier la quête de l'ailleurs des migrants économiques, les traversées des exilés, la conquête du 
territoire de l'Autre. Le cas de Vourbié illustre bien l'exil forcé dans la mesure où il est expulsé sans pitié du village par «le peuple qui ne traverse jamais le fleuve » pour avoir transgressé doublement les lois de la tradition :

Depuis la préparation des funérailles du Doyen, un vent d'horreur et de violence soufflait sur le village des Incirconcis [...] Il naquit un enfant de la dernière femme de Vourbié, qui porta le nombre de ses descendants à vingt-sept. Mais cet enfant était particulier et souleva, comme le vent aux origines et aux destinations multiples, des questions qui jusqu'à ce jour, préoccupent encore le conseil des Anciens. En effet, dans la millénaire tradition des Incirconcis, naquit cet enfant avec une différence notable que personne n'osa appeler anomalie ; il naquit circoncis! (Bazié LTN 165)

Baki et Elema ont également franchi à leur détriment les limites de l'interdit, en pénétrant le bois sacré en pleine cérémonie d'initiation. Elema en fait les frais :

Elema ne voit plus rien et court aveuglément dans l'obscurité. Elle trébuche violemment contre une racine, s'étale de tout son long sur les feuilles mortes, les branches sèches et le sol mou, dans un grand bruit. Le masque atterrit l'instant d'après sur elle, et il s'engage un corps à corps sans merci entre la femme et l'homme-animal.[...] Ce deuxième deuil, occasionné par la mort d'Elema découverte après la poursuite, a aussi donné une fin plus tragique aux festivités et contribué à soulever plusieurs questions que nul ne peut élucider. (Bazié LTN 217, 223)

En remettant en question les frontières, Bazié pousse à réfléchir sur la notion d'étranger, d'autochtone, de déconstruction identitaire. Dans cette optique, on n'est pas bien loin de «l'illusion de l'altérité » dont parle Bernard Mouralis ${ }^{16}$. Cette illusion emprunte plusieurs visages ici relativement à la souffrance individuelle : l'illusion traditionnelle (rêve illusoire de garder intact la tradition); l'illusion transgressive (aspiration illusoire au dépassement des frontières); l'illusion de transitivité (désir chaotique et aléatoire du voyage hors du cadre familier); l'illusion de sécurité (rêve d'occupation d'une zone permanente de confort); l'illusion de richesse (aspiration quasi-onirique à l'accumulation de biens matériels). Avec 147 mots dans le roman caractérisant l'obscurité, on peut entrevoir qu'il s'agit des utopies traduisant la complexité des itinéraires identitaires et la quête de lumière.

L'éloignement physique des origines est compensé par un retour à travers le souvenir et l'émotion. Il s'agit là d'un processus historique dont la fluidité féconde la création littéraire des "migrant writers ». Contrairement aux autres écrivains qui résident au Québec comme exilés/migrants et dont la coprésence de plusieurs langues influence leurs écrits, Bazié s'attache à un français limpide, dans sa nouvelle

\footnotetext{
16 On peut consulter avec bonheur, Bernard Mouralis, L'Illusion de l'altérité. Etudes sur la littérature africaine, Paris, Honoré Champion, 2007. Dans cet important ouvrage, Mouralis note que la production littéraire de l'Afrique apparaît d'abord comme un immense questionnement, multiforme, qui s'opère dans un cadre qui ne correspond pas nécessairement aux frontières tracées par la critique et la recherche et qui porte moins sur la définition de l'identité africaine que sur la définition opérée par un sujet, d'une relation : entre les peuples, les époques, les langues et les littératures.
} 
patrie qu'est le Québec ${ }^{17}$. Il se l'approprie très bien pour se faire mieux comprendre, $\mathrm{s}^{\prime}$ affirmer et s'assumer. Autrement dit, la langue agit pour lui comme le premier agent de déterritorialisation. Ce dernier terme mérite que nous lui portions ici une attention toute particulière, en lien avec l'utopie et la question de la citoyenneté.

\section{Utopie, écarts identitaires, citoyenneté et déterritorialisation}

L'écriture des « expatriés culturels » africains investit des espaces multiples, signe de l'itinéraire instable des écrivains. Chez ceux-ci, le territoire d'énonciation du texte littéraire remet en question la notion d'étranger et d'autochtone, la frontière entre le centre et la périphérie leur permettant de se reconnaître dans une identité multiculturelle. De plus, l'innovation esthétique et l'invention littéraire transparaissent dans cette littérature qui s'écrit aux charnières des cultures et est empreinte d'interculturalité. Comme pour le texte programmatique de Thierry Fabre Traversée (2001), le titre du roman LTN renvoie à l'idée de transbordement, de passage, de franchissement. La tonalité utopiste et idéaliste de l'œuvre débouche sur une aporie, et c'est probablement ce qui justifie le qualificatif «nocturne » accolé au titre. En disant le pays étant ailleurs, un décalage entre cet ailleurs et la réalité concrète des paysages signale une sorte de mirage, $\mathrm{du}$ fait d'un nouvel espace identitaire dont les frontières font éclater les cadres ordinaires. De ce point de vue, les scènes chez Bazié se déroulent tantôt en Afrique, tantôt en Allemagne ou en France. On y évoque même les États-Unis, alors que l'auteur lui-même réside au Canada. Le passage suivant illustre cette mobilité spatiale, tout autant que le choc interculturel qui en découle: «Et c'est ainsi qu'un jour, une jeune Blanche amena Drabo en Allemagne (...) Une fois en Allemagne, Drabo se rendit compte d'un premier obstacle à ses ambitions, la langue (...) et quelques mois plus tard, il se retrouva en France. » (Bazié LTN 141- 142)

L'appartenance réelle ou symbolique ici ne découle pas du concept pays/patrie. Il $\mathrm{s}^{\prime}$ agit là d'un mode différent d'autoreprésentation de la communauté diasporique. Ce qui confronte, en bonne logique, la question d'identité à celle de la citoyenneté.

Si la citoyenneté est le trait caractéristique de toute personne qui, habitant une cité à laquelle elle appartient et y a droit au suffrage dans les assemblées publiques, l'identité, elle, renvoie au sentiment pour un individu d'appartenir à une culture donnée. La citoyenneté et les identités sont variables selon que l'on se retrouve en

\footnotetext{
${ }^{17}$ Par exemple, Jérôme Ceccon analysant les formes italiennes et dialectales chez les écrivains italoquébécois, relève que : «les écrivains italo-québécois qui écrivent au Québec ont souvent choisi le français comme langue d'écriture. Toutefois, la plupart ont été élevés à l'école anglophone et parlaient un dialecte à la maison. Ils se retrouvent donc avec plusieurs langues dans leur vie et cela se voit dans leurs écrits. » (Ceccon 193)
} 
territoire urbain ou en territoire rural. Pour s'affirmer comme citoyen d'un village africain, il faut accepter et respecter les règles qui y sont établies par la tradition. La citoyenneté s'applique à l'individu par un ensemble de devoirs à assumer (Munzangala-Munziewu 81-93). C'est pourquoi le père de Vourbié dans LTN demande à être enterré au centre de Kiekena. Le refus du fils d'exécuter à la lettre cette dernière volonté attire sur lui le rejet de la communauté toute entière. Le sort l'amènera à engendrer un fils déjà circoncis, contrairement à la tradition qui soumet la circoncision des garçons à un rituel initiatique. En effet, la citoyenneté et l'identité à Katena s'affirment par le passage de rites d'initiation, difficile et secrète. Ces rites marquent l'entrée des jeunes dans la caste des «porteurs de masque ». Ces rites sont placés sous la houlette de trois maîtres masqués, difficiles à identifier. Ils se déroulent tous les trois ans, pendant trois jours dans le bois sacré. Ils se clôturent par une fête associant toute femme se reconnaissant citoyenne de Katena. Elle doit participer aux festivités par la cuisson du «Dolo de sorgho » consommée pendant la cérémonie marquant la fin de l'initiation. Tout refus de collaboration comme c'est le cas du vieux Baki, ancien combattant retraité vivant à Katena, constitue une hérésie. Baki a non seulement construit sa maison loin du grand cercle de son clan, mais se fait craindre de tout le village par son fusil et son invulnérabilité au plan occulte.

Vourbié, Baki et son épouse Elema ont une citoyenneté complexe - ou encore ambiguë pour les leurs - parce qu'ils n'arrivent pas à se conformer aux lois établies, à remplir leurs devoirs coutumiers vis-à-vis de la communauté villageoise. La transgression des interdits ici les rend passibles de mort ou d'exil. En découvrant l'identité réelle du porteur de masque, Baki et Elema se sont exposés à des représailles sans pitié. Lorsque l'épouse de Baki s'aventure dans la forêt sacrée pendant l'épreuve d'initiation, elle viole un tabou pour entrer dans le «secret absolu ». Le croisement de son regard avec celui du masque sonne le glas de son appartenance à la communauté de Katena, de sa citoyenneté.

En revanche, dans les centres urbains, d'autres valeurs déterminent la citoyenneté : le cosmopolitisme, les papiers officiels et les charges financières. La ville est le lieu où cohabitent des élites venues de divers horizons et de statuts différents. Dans LTN le frère $\mathrm{du}$ capitaine Démocrate visite le Pentagone: milieu des intellectuels d'un certain niveau d'étude. Il ne peut réussir dans ce milieu comme Alice, Edmond, et bien d'autres intellectuels de la zone. D'où leur citoyenneté et leurs identités complexes. Au-delà de ce cosmopolitisme, la vie en ville exige une citoyenneté régie par la possession de la carte d'identité nationale, le passeport et autres cartes subsidiaires. L'absence de possession de ces pièces fait perdre l'identité et, par conséquent, sa citoyenneté. Comme le dit Jean-Claude Ruano- Borbalan,

Pour les immigrés, la construction identitaire est une dynamique incessante de confrontation aux valeurs dominantes de la société d'installation et d'affirmation de leur propre valeur individuelle. Face aux injonctions contradictoires entre la culture d'origine et la culture d'accueil, plusieurs attitudes sont observées. La majorité des immigrés fuit la contradiction en 
adoptant la culture d'accueil. Enfin, certaines attitudes, elles aussi minoritaires, consistent à vivre une totale séparation entre une morale ancrée sur les valeurs traditionnelles de la culture d'origine et la vie quotidienne. (Ruano-Borbalan 5)

L'utopie de l'exilé, dont le départ est vu comme une libération, peut être envisagée comme un potentiel échec. Comme dans le roman d'Alain Mabanckou Les Petits-fils nègres de Vercingétorix (2002), l'expérience de l'étrangeté, de l'aliénation place les personnages dans une situation de tourmente et d'insécurité identitaire, de conscience déchirée, parce que le point d'attache devient flou sans qu'il n'y ait possibilité de retourner. Il y a mise en échec d'une conception identitaire non exclusive et figée.

L'écriture de l'utopie constitue, pour ainsi dire, le versant de la déconstruction ${ }^{18}$, de la déterritorialisation, fonctionnant comme une «castration symbolique ». Cette dernière «nous enseigne(nt) en tout premier lieu qu'une seule vision de la réalité, une seule étiquette ne suffisent jamais pour rendre justice à la complexité de l'être humain et de son monde. Elle nous permet de percevoir dans l'autre non seulement l'écart d'avec notre propre vision du monde mais aussi les parallèles et ressemblances. » (Matis-Moser 236).

\section{Conclusion}

Finalement, chez Isaac Bazié comme chez les autres écrivains exilés/migrants africains, l'errance est mise en actualité par l'écriture de l'utopie, même si l'espace rêvé génère une souffrance liée à l'absence de maîtrise des codes sociaux dans des filiations multiples ${ }^{19}$. Le questionnement identitaire, la dérision des convictions sociales figées, la parodie des personnages impuissants face à leurs propres lois, l'exil investissent l'espace de la création littéraire de la communauté diasporique. Nous avons pu observer que du tiraillement identitaire naît une ambiguïté culturelle contradictoire au rêve initial, faisant de l'écriture une quête sans fin. Le cosmopolitisme, au-delà du rêve brisé, crée un nouvel espace de rêve, là où les traditions meurtries constituent les facteurs majeurs de la déterritorialisation comme l'a montré Bazié à travers son roman. Ce dernier mène une réflexion sur la superficialité d'une réalité et la représentation esthétisée par une pluralité de références culturelles, informatives et sensorielles. Chez Bazié, pas d'engagement frontal ni mission littéraire, mais une migration à la fois du promoteur et du littéraire. La circulation du texte entre divers lieux et diverses générations et catégories sociales génère une écriture migrante caractérisée par l'utopie et les angoisses de l'errance/fixité. L'exil n'est finalement pas simplement géographique, il

\footnotetext{
${ }^{18}$ Littérature du déracinement ou de déconstruction identitaire (Chevrier 96-100) caractérise ainsi la littérature de l'immigration.

19 Le rattachement de l'écrivain francophone africain à plusieurs cultures souligne son multiculturalisme, alors que dans le même temps, l'interculturalité le met en rapport à d'autres cultures.
} 
http://ejournals.library.ualberta.ca/index.php/af

est l'expression d'un réel manque fécond qu'on nourrit le rêve de combler par l'écriture de l'entre-deux identitaire, dans le cadre de la littérature francophone. 


\section{Bibliographie}

- Albert, Christiane. L'immigration dans le roman francophone contemporain. Paris: Karthala, 2005.

- Bazié, Isaac. La Traversée nocturne. Ottawa/Ontario : Malaika, 2004.

- Bazié, Isaac. «Roman francophone : écriture, transitivité, lieu. » Tangence 75 (été 2004) :123-137.

- Bhabha, Homi. Les Lieux de la culture ; une théorie postcoloniale. Paris : Payot, 2007.

- Ceccon, Jérôme. « Migration et identité: l'exemple italo-québécois. » Francofonia 13 (2004) : 193-207.

- Chevrier, Jacques. «Afrique(s)-sur-Seine, autour de la notion de migritude. » Notre Librairie 155- 156 (2006) : 96-100.

- Clavaron, Yves. "La mise en scène de l'altérité dans la littérature postcoloniale: entre insécurité et hybridité. » Ethiopiques 74 (2005) : 105-118.

- Diop Papa samba et Paravy, Florence (dir) Littératures africaines. Littératures francophones et utopies. Paris : Université Paris XII Val de Marnes, 2006.

- Derrida, Jacques. Le Monolinguisme de l'autre. Paris : Galilée, 1996.

«Entre-deux/in-between; Bordeline ». Dictionnaire international des termes littéraires.

Ed. Grassin Jean-Marie, Projet initié par l'Association de Littérature Comparée. 8 septembre 2009 http://www.ditl.info/arttest/art1546.php

- Feze, Yves-Abel. «Les littératures francophones ont-elles une identité ?» Interculturel 11 (2007) : 243-253.

- Gbanou, Sélom Komlan. «Le fragmentaire dans le roman francophone africain. » Tangence_75 (2004) : 83-105.

- Genette, Gérard. Introduction à l'architexte. Paris : Seuil, 1979.

- Halpern, Cathérine et Jean-Claude Ruano-Borbalan Identité(s). L'individu. Le groupe. La société. Paris : Ed. Sciences Humaines, 2004.

- Imbert, Patrick. Trajectoires culturelles transaméricaines. Médias, publicité, littérature et mondialisation. Ottawa : Les Presses de l'université d'Ottawa, 2004.

- Kom, Ambroise. La malédiction francophone. Défis culturels et condition postcoloniale en Afrique. Hambourg : Lit Verlag/Clé, 2000.

- Kristeva, Julia. Sémiotikè. Recherches pour une sémanalyse. Paris : Seuil, 1969.

- Laronde, Michel (dir). L'Écriture décentrée. La langue de l'Autre dans le roman contemporain. Paris : L'Harmattan, 1997.

- Lopes, Henri. Dossier classé. Paris : Seuil, 2002.

- Maingueneau, Dominique. Le Discours littéraire: paratopie et scène d'énonciation. Paris : Armand Colin, 2004.

- Mathis-Moser, Ursula. «La littérature française: une littérature qui fait la différence ? » Dialogues et cultures 53 (2008) : 229-236.

- More, Thomas. L'Utopie. Flammarion : Paris, 1972.

- Moura, Jean-Marc. Littératures francophones et théories postcoloniales. Paris : PUF, 1999.

- Mouralis, Bernard. L'Illusion de l'altérité. Etudes sur la littérature africaine. Paris : Honoré Champion, 2007. 
- Munzangala-Munziewu, Dieudonné. «Entre citoyenneté et ethnicité : perspectives africaines d'une identité démocratique. » Palabres actuelles 1 (2007) : 81-93.

- Ollivier, Émile. «Et me voilà otage et protagoniste» Boutures 1.2 (2000) : 22-26.

- Pangop, Alain. «L'écriture du trauma postcolonial en Afrique subsaharienne.» Littérature et déchirures. Ed. Dili Palaï et Daouda Paré. Paris : L'Harmattan, 2008. 105122.

- Ranvier, Alain. «Exil. » Dictionnaire du littéraire. Ed. P Aron et alii. Paris : P.U.F., 2002. 205-207. 